\title{
An Analog Circuit Fault Diagnosis Method Based on SVM Optimized with BQPSO
}

\author{
Wang Zhongsheng, a , Yang Sen ${ }^{2, b}$ and Huang Shujuan ${ }^{1, a}$ \\ ${ }^{1}$ School of Computer Science and Engineering, Xi'an Technological University, Xi'an \\ 710021, China, \\ 2Department of UAV Engineering, Ordnance Engineering College, Shijiazhuang, China, \\ a59483672@qq.com, b568657132@qq.com
}

Keywords: BQPSO; SVM; Analog circuit fault diagnosis.

\begin{abstract}
In order to improve the correct rate of analog circuit fault diagnosis, a method based on SVM optimized with BQPSO is put forward. Firstly, BQPSO algorithm and its steps are presented; Then the performance impact factors of SVM are analyzed, and the steps of SVM parameters optimized with BQPSO are given; Finally, a filter circuit is taken as an example to simulate. The result shows that this method is effective.
\end{abstract}

\section{Introduction}

Due to the lack of a simple fault model in analog circuit, component parameters' tolerance and the influence of noise make the analog circuit fault diagnosis very complex. It also restricts the use of traditional fault dictionary method, Bayesian method, and the case-based reasoning method. Artificial Neural Network (ANN) has lots of fine features, such as massively parallel distributed processing, associative memory, self-organization learning, robustness, fault tolerance and so on. It also has a strong processing capacity for uncertain knowledge, able to solve many problems that the traditional methods can not solve. ANN, however, has some disadvantages, such as complex network structure and other issues.[1][2].

SVM is a new machine learning approach developed from the statistical learning theory which is especially used for the search for the theory of machine learning on the condition of small sample. The kernel of statistical learning theory is that it coordinates the contradictions between learning capacity and generalization capacity with the choice of structural risk minimization. SVM overcomes the deficiencies of neural network and it presents the advantages of simple structure, global optimization and excellent generalization capacity in solving problems of small sample, nonlinear and high dimensionality pattern recognition[3][4].

It is very important to complete the parameters optimization of SVM. There is not a best accepted solution about the parameters optimization. The usual method is to select parameters with crossing validation. However, it is low efficiency and poor precision. Aiming at solving these problems, this paper gives an analog circuit fault diagnosis method based on SVM optimized with Binary-coded quantum particle swarm optimization (BQPSO). BQPSO algorithm is firstly introduced in this paper, and the SVM parameters which influence the classification performance are analyzed. Then SVM parameters are optimized using BQPSO algorithm. Finally, the validity of the proposed method is verified according to the example of analog circuit fault diagnosis.

\section{BQPSO Algorithm Analysis [5][6]}

In order to make the quantum particle swarm optimization algorithm solving discrete searching space problem in actual problems, the binary-coded quantum particle swarm optimization algorithm (Binary QPSO, BQPSO) is introduced.

The dimension of particle is defined as the number of decision variables contained in particle,

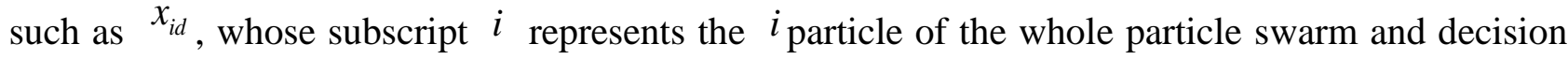


variable $^{d}$ represents $x_{i d}$ dimension of $d$. The length of $x_{i}$ and $x_{i d}$ are represented by $l$ and $l_{d}$. Then:

$$
\begin{aligned}
& l=\sum_{i=1}^{d} l_{d}, d=1,2, \cdots D \\
& D \text { is the number of decision variables. }
\end{aligned}
$$

The distance between two particles $x_{1}$ and ${ }^{x}$ can be represented by Hamming distance:

$$
\left|x_{1}-x_{2}\right|=d_{\text {Hamming }}\left(x_{1}, x_{2}\right)
$$

In BQPSO algorithm, Generation mode of attractor $p_{i}$ is similar to the crossover in genetic algorithm. It is produced by randomized crossover of $P_{i}$ and $P_{g} . p_{i}$ meets the Hamming distance defined by two equations above.

The method of producing new location by crossover method in this paper is defined as a mutation test. Mutation probability $p_{v}$ is defined as:

$$
p_{v}= \begin{cases}\alpha \cdot d_{\text {Hamming }}\left(\text { mbest }_{d}, x_{i d}\right) \cdot \ln (1 / \mu) & \text { if } \alpha \cdot d_{\text {Hamming }}\left(\text { mbest }_{d}, x_{i d}\right) \cdot \ln (1 / \mu)<1 \\ 1 & \text { otherwise }\end{cases}
$$

$\mu$ is random number between 0 and 1 . Mutations of every $p_{i}$ position are decided by $p_{v}$. BQPSO algorithm steps are as follows:

Step1: Initialize the particle swarm in binary form, and calculate the particle fitness value.

Step2: Average calculation of discrete particle pbest.

Step3: Calculate local attractor of the particle by intersecting.

Step4: Calculate new particle by mutating.

Step5: Update pbest.

Step6: Update gbest.

Step7: Repeat steps Step2 Step6, until meet stop condition of the algorithm.

BQPSO algorithm has the advantages of simple operation, realized convenience, fast convergence, less controlled parameters and so on. Compared with traditional PSO algorithm, it has stronger search performance [7].

\section{Analysis of the Impact Factor of SVM[7]}

The factor that affects the prognostic capability of SVM includes: kernel function and its parameter, punishment coefficient $\mathrm{C}$ and insensitive loss coefficient $\mathcal{\varepsilon}$.

Kernel Function and Parameter. Kernel function, mapping function and characteristic space are corresponding to each other, making sure kernel function means making certain mapping function and characteristic space. The change of kernel function's parameter could actually impact the complicated degree of the sub-space for changing a sample data. The dimension of the data sub-space comes to a decision that the ability to categorize the biggest VC dimension of surface in sub-space structure line limits the complicated degree of the superior classification surface, which can be constructed in data sub-space and also came to a decision that the minimum experience risk of the line classification surface can be attain.

The kernel function in SVM could be chosen in line kernel function, polynomial kernel function, $\mathrm{RBF}$ kernel function and sigmoid function etc. Contrasted with RBF kernel function, the line kernel is only a kind of particular example of RBF kernel, RBF kernel function can be also used in 
non-linear sample to reflect high dimension; The parameters of polynomial kernel function are more(RBF kernel function only has one parameter $\gamma$ ) than that of RBF kernel function, the model choice is getting more complicated, in the meantime when count is higher, it is larger for computing complications; Sigmoid kernel function will cause an illegal value under some certain parameters. Therefore, in majority case of circumstances, RBF kernel function is a better choice.

Punishment Coefficient $\mathbf{C}$. The function of punishment parameter $\mathrm{C}$ is to compromise the margin of structure risk and sample error, also make the model have a better expansion ability. The punishment to the sample of miscarriage of justice will be small in the sample data. When the training error margin becomes large, the expansive ability of the system becomes bad, then it leads to the "owe study" phenomenon; The parameter C leads to study's accuracy corresponds precise and the model approaches minimum actual experience risk, however the model's suffused ability of turning becomes bad, which will appear "over study" phenomenon. Moreover, the C value influences the processing of isolated point in the sample and selecting suitable $\mathrm{C}$, which can promise anti-interference to some extent and ensure the stability of the model .

\section{Optimize the SVM Parameters Based on BQPSO}

The concrete steps of BQPSO arithmetic to optimize SVM parameters are as follows:

Particle Coding. For class imbalances, two types are punished by two penalty parameters, as shown below:

$$
\begin{array}{ll}
\min _{\psi, b} & \frac{1}{2}\|\psi\|^{2}+C_{+} \sum_{y_{i}=1} \xi_{i}+C_{-} \sum_{y_{i}=-1} \xi_{i} \\
\text { s.t. } & y_{\mathrm{i}}\left(\left(\varphi\left(x_{i}\right)^{*} \psi\right)+b\right) \geq 1-\xi_{i} \\
& \xi_{\mathrm{i}} \geq 0, i=1,2, \cdots, l
\end{array}
$$

In the equation, $C_{+}, C_{-}$are different types of penalty parameters.

The following formula is as follow:

$$
\frac{C_{+}}{C_{-}}=\frac{N_{-}}{N_{+}}
$$

In the equation, $N_{+}, N_{-}$is the number of different class samples.

In the concrete use, we could only consider optimizing $C_{+}$, and then use $C_{+}$to calculate $C_{-}$ through (5). Once two categories' data qualities are equal, $C_{+}, C_{-}$are equal, all of which are consistent with the normal C.

Particle code can be defined by the above analysis. SVM parameters are real numbers, but type judgment requires discrete codes, so particles are encoded by discrete method. Particle consists of two parts, penalty parameter $C_{+}$section and kernel parameters section.

The first part uses a binary bit string to represent penalty parameter $C_{+}$, and then uses the following formula to convert the binary bit string to specific actual parameters in decimal.

$$
C_{+}=\frac{C_{+\max }-C_{+\min }}{2^{l_{C_{+}}}-1} \times d_{C_{+}}+C_{+\min }
$$

In this equation, $C_{+}$is a decimal value, $C_{+\min }$ represents the minimum value of $C_{+}, C_{+\max }$ represents the maximum value of $C_{+},{ }_{C_{+}}$represents the binary bit string length of $C_{+}, d_{C_{+}}$represents the decimal value of the bit string of $C_{+}$. Precision of parameter is determined 
by the length of the bit string representing the parameter; range is determined by the minimum and maximum parameters, both of which can be set as desired.

The second part represents kernel parameters. When using RBF kernel function, it represents $\gamma$. The conversion formula is similar to (6):

$$
\gamma=\frac{\gamma_{\max }-\gamma_{\min }}{2^{l_{\gamma}}-1} \times d_{\gamma}+\gamma_{\min }
$$

In this equation, $\gamma$ is decimal value, $\gamma_{\min }$ represents the maximum value of ${ }^{\gamma}{ }{ }$ represents the binary bit string length of $\gamma, d_{\gamma}$ represents the decimal value of the bit string of $\gamma$.

Total length of particle $l_{x}=l_{k}+l_{C_{+}}+l_{\gamma}$

Determine the Fitness Function. Support vector machine is used to calculate cross-validation accuracy for each node on the training set of ten-fold, as for node category, support vector machine optimization parameters is selected on the basis .

Set Up Elitist Set. For a specific optimization target, the optimal solution is often more than one. In the mechanism of basic discrete particle swarm algorithm, the global optimum particle is only one. In BQPSO algorithm an elitist set is defined for storing a plurality of possible optimal solution, if in the same iteration, while there are many particles which have maximum fitness and the fitness is greater than the fitness of particles in elitist set, then all of the iterative optimal particles are stored in elitist set, the original particles of elitist set are removed. Obviously, when the algorithm ends, all particles in elitist set are the optimal solutions of the problem. For the optimal solutions obtained, they are supposed to train support vector machines one by one, calculate the total number of support vector, and select the least total as the last best solution.

\section{Analog Circuit Simulation Application}

The efficiency of the algorithm could be validated by some low-pass filter circuit examples. Low-pass filter circuit is shown in Fig. 1, and the amplitude-frequency response in normal state is shown in Fig. 2.

Assuming the circuit has single soft faults, we extracts the amplitude of each frequency point in [ 1KHZ,1MHZ], and samples every ten times frequency sampling 10 points . As shown in Fig. 1.

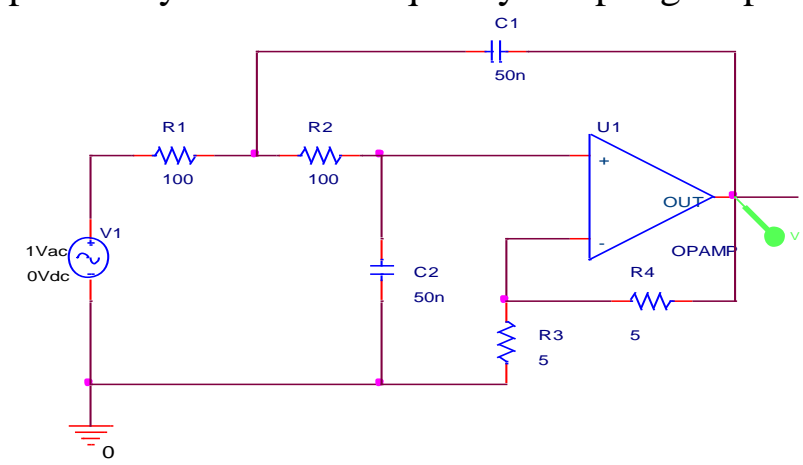

Figure 1. Low-pass filter circuit 


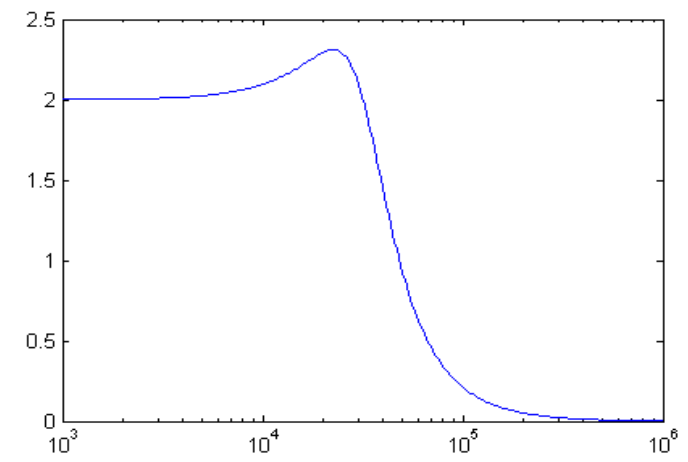

Figure 2. Amplitude-frequency response of low-pass filter circuit

Table.1 Kinds of single soft faults

\begin{tabular}{|c|c|c|c|}
\hline Fault No. & Fault class & Fault No. & Fault class \\
\hline F1 & $\mathrm{NF}$ & F2 & $C_{1}=2 \sin ^{2}$ \\
\hline F3 & $C_{1}=75_{n} F$ & F4 & $C_{2}=25 x I$ \\
\hline F5 & $C_{2}=75 x$ & F6 & $R=15 \Omega$ \\
\hline F7 & $R_{1}=50$ & F8 & $R_{2}=15 \Omega$ \\
\hline F9 & $R_{2}=5 \Omega$ & F10 & $R_{3}=7.5 \Omega$ \\
\hline
\end{tabular}

The circuit had already been simulated 200 times by Monte Carlo in the case of normal state and the various fault states. Their fault features are sampled, which could be divided into two parts.

Respectively, SVM parameters are selected by means of the traditional cross validation method and the proposed method in this paper, in order to determine the best parameter values. Finally, obtained results of classification accuracy in the test set are shown in Table 2.

Table.2 Comparison of test set classification results

\begin{tabular}{cc}
\hline $\begin{array}{c}\text { Parameter optimization } \\
\text { method }\end{array}$ & Classification accuracy \\
\hline 10-fold cross-validation & $85.33 \%$ \\
proposed method in this paper & $95.71 \%$ \\
\hline
\end{tabular}

As can be seen from the above results, analog circuit fault diagnosis method based on SVM Optimized with BQPSO is significantly higher than the traditional method on the classification accuracy .It also can effectively improve the precision of analog circuits fault diagnosis, which has important practical significance.

\section{Conclusion}

On the basis of analyzing the SVM effective factors, SVM parameters could be optimized with BQPSO algorithm. After taking a low-pass filter circuit as the research object to make a simulation and comparing with the tradition cross validation method, the effectiveness of the method in this paper is verified.

\section{Acknowledgment}

This paper is supported by the Special Scientific Research Project of Shaanxi Province Education Department (NO. 15JK1342), and supported by the Department of Education special fund of Shaanxi Province [2013] 23 


\section{Reference}

[1] V. Sugumaran, V. Muralidharan, K. I. Ramachandran.Feature selection using Decision Tree and classification through Proximal Support Vector Machine for fault diagnostics of roller bearing [J].Mechanical Systems and Signal Processing, 2007, 21: 930-942.

[2] Tai-Yue Wang, Huei-Min Chiang.Fuzzy support vector machine for multi-class text categorization [J].Information Processing and Management, 2007, 43: 914-929.

[3] Allwein E L, Schapire R E, and Singer Y.Reducing Multiclass to Binary: A Unifying Approach for Margin Classifiers [J].Journal of Machine Learning Research, 2000, (1): 113-141.

[4] DUAN Xiu-sheng, SHAN Gan-lin.Hierarchical Support Vector Machine for Multi-class Classification [J]. JOURNAL OF ORDNANCE ENGINEERING COLLEGE, 2009, 21(1): 64-66.

[5] Li Baochen, Huang Kaoli. Study on Generation of A Multiple Fault Minimal Candidates Set Based on BQPSO [J]. Computer Measurement \& Control, 2013, 21(6): 1476-1482.

[6] HAN Ying-xian, LIU Jing. Path Planning Algorithm for Underwater Vehicle Based on BQPSO [J]. COMPUTER ENGINEERING, 2011, 37(8): 216-218.

[7] Wang Cheng, Yang Sen. Research on Fault Prediction of Power Supply Based on SVR Optimized with Improved QPSO [J]. Computer Measurement \& Control, 2014, 22(5): 1342-1344. 